\title{
The application of Fourier transform to the identification of temperature distribution in HFE-7100 flow boiling in an annular minigap
}

\author{
Sylwia Hożejowska ${ }^{1, *}$, and Magdalena Piasecka² \\ ${ }^{1}$ Kielce University of Technology, Faculty of Management and Computer Modelling, Al. 1000-lecia PP 7, 25-314 Kielce, Poland \\ ${ }^{2}$ Kielce University of Technology, Faculty of Mechatronics and Mechanical Engineering, Al. 1000-lecia PP 7, 25-314 Kielce, Poland
}

\begin{abstract}
In the paper the results of investigations into HFE-7100 flow boiling heat transfer in a cylindrical minigap a $1 \mathrm{~mm}$ wide created between the external glass pipe and the copper pipe were discussed. A cartridge heater located axially heated fluid flowing along the minigap. The cooling fluid temperature and pressure at the inlet and the outlet to/from the minigap and temperature of the heater in 18 points were measured. Two-dimensional mathematical model for heat transfer coefficient determination was proposed. It was assumed that in the test section the heat transfer process was in steady state and the fluid flow was laminar. The temperature of the metal pipe near the heater was assumed to satisfy Laplace's equation. The problem formulated in this way was solved by two methods: the Fourier transform and the Trefftz method. The working fluid temperature was calculated depending on the flow type: for single phase flow with boiling incipience and for two-phase flow, respectively. The heat transfer coefficient at the fluid copper pipe interface was calculated due to the Robin condition. Local heat transfer coefficient values obtained from the Fourier transform and from the Trefftz method were similar. Results were presented and discussed.
\end{abstract}

\section{Introduction}

Heat exchangers with minigaps have attracted wide attention from the heat transfer issues due to their prominent ability to remove heat flux from confined spaces. Highly efficient heat transfer system is of great importance to the operations and performance of high power components of heat exchangers. Miniaturization of heat transfer exchangers help to decrease size of these devices.

Recently, a lot of works have been published to address the issues of heat transfer in flow boiling in minigaps of different geometry. Previous authors' studies mostly have been performed to evaluate the flow boiling heat transfer in rectangular minichannels, as [1-5]. This paper shows the results of research on flow boiling heat transfer during refrigerant flow along an annular minigap [6-8]. Cylindrical geometry of a minigap in other researchers' works can be find in [9-11].

Currently, the research often abandons the experimental studies in favour of numerical simulations, utilising commercial software, where the experimental results serve the purpose of validation of numerical calculations only, such as, for example, in $[12,13]$. Application of commercial software for solving an inverse heat conduction problem (IHCP), as contemplated in this paper, is limited, which results from the following facts:

- too small set of available experimental data which have to be implemented into the codes,

- necessity to solve instantaneously energy equations in the annular minigap and the heated surface with an incomplete set of closure equations (unknown boundary condition on the unheated side of the channel).

In this paper, two methods are used for solving the IHCP: the Fourier transform and the Trefftz method [8].

\section{Experimental background}

The schematic diagram of the experimental stand is presented in Fig. 1.

The flow loop in which a working fluid (HFE-7100) circulates and the data acquisition system consists of: the test section (1), a tube-type heat exchanger (5), a compensating tank (6), a deaerator (7), a filter (8), a gear pump (9) and a mass flow meter (10). The data acquisition system comprises: two data acquisition stations (2, 3), a pc computer (4) with a specialist software and a high speed camera.

The schematic diagram of the test section is shown in Fig. 2. A minigap (3) $1 \mathrm{~mm}$ wide was created between a glass pipe (1) and a copper pipe (2) positioned along the same axis. A cartridge heater (4) was located inside

\footnotetext{
*Corresponding author: ztpsf@tu.kielce.pl
} 
the pipe axially. To fix thermoelement sensors (6) with a spacing of approximately $10 \mathrm{~mm}$ in the flow line longitudinal slots was made in the inside surface of the pipe. Thermoelement wires run in the gap between the pipe and the heater in the thermal paste. The sensors of thermoelements to measure the working fluid temperature (6) and pressure meters (7) for fluid pressure control at the inlet and the outlet to/from the minigap, were placed in the module headers (5).

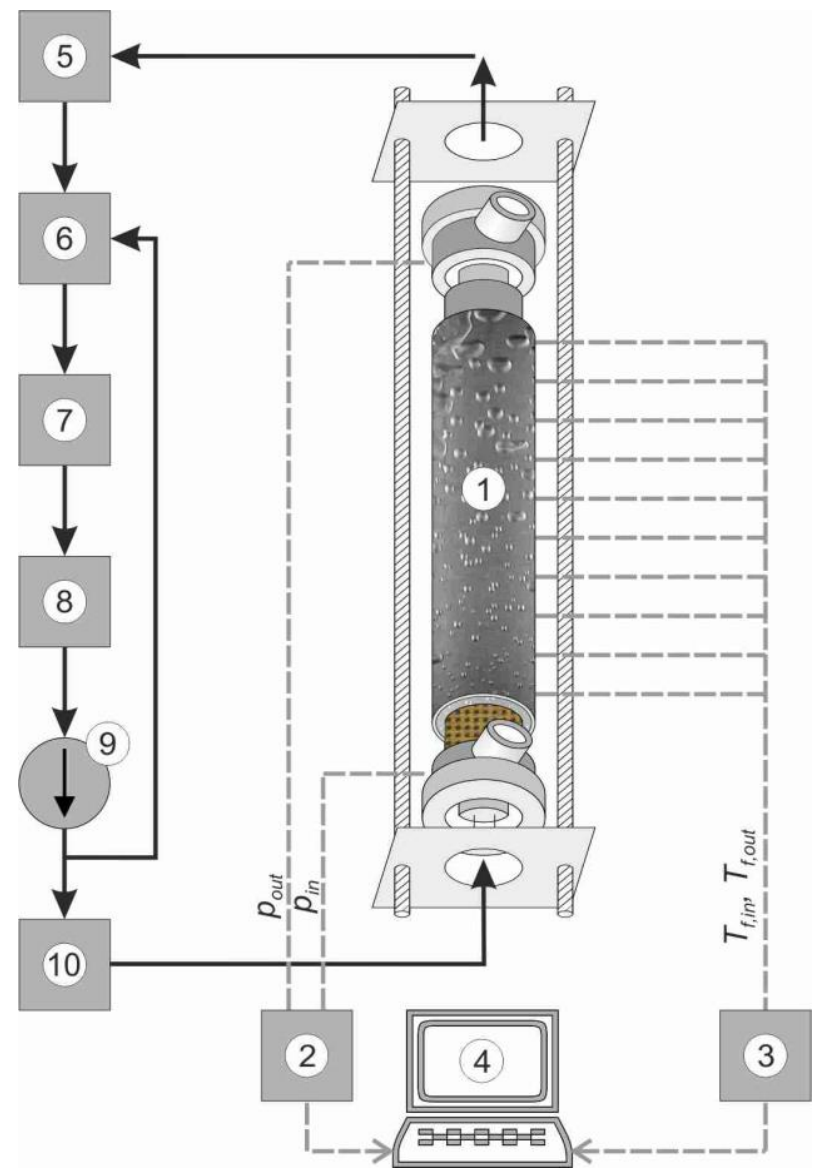

Fig.1. The schematic diagram of the experimental stand: 1 - the test section, 2,3 - data acquisition stations, 4 - a pc computer, 5 - a tube-type heat exchanger, 6 - a compensating tank, 7 - a deaerator, 8 - a filter, 9 - a gear pump, 10 - a mass flow meter.

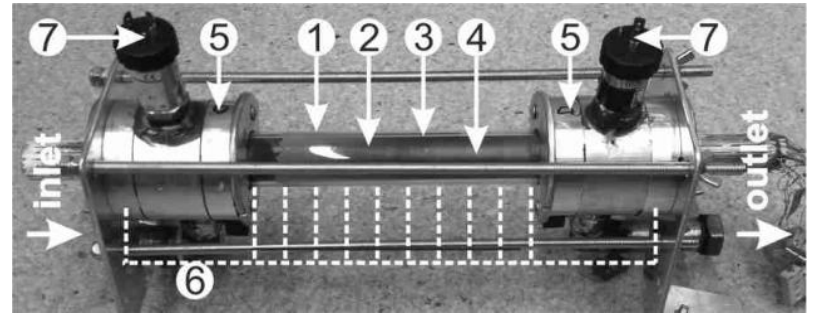

Fig. 2. The schematic diagram of the test section: 1 - a glass pipe, 2 - a copper pipe, 3 - a minigap, 4 - a cartridge heater, 5 - a module header, 6 - thermoelements, 7 - a pressure meter.

During the experiment, there was a HFE-7100 (3M) flow in the flow loop along the minigap. The heat flux supplied to the heater in the test section was increased gradually, by adjustment of heating power by autotransformer with controlled current intensity, until developed nucleate boiling occurs. The signals from the thermocouples and the pressure meters, the power supplied to the heater and mass flow rate were recorded by data acquisition stations.

\section{Mathematical model and numerical solution}

In a two - dimensional mathematical model it was assumed the steady state in the test section and the laminar flow of the fluid (Reynolds number $<2100$ ).

Scheme of the test section is presented in Fig. 3. The heat transfer process in the test module is axisymmetric i.e. the temperature distribution of the elements in the module is only dependent on two variables: $z$ (along the flow direction) and $r$ (referring to the thickness of the cartridge heater and the copper pipe). It was assumed that the thin layer of the thermal conductive filler $\left(\sim 10^{-4} \mathrm{~m}\right)$ and the size of the thermocouples placed in it were omitted. Additionally, it was assumed that the surface has direct contact with the surface of the heater. All the thermal properties were stationary and independent on temperature.

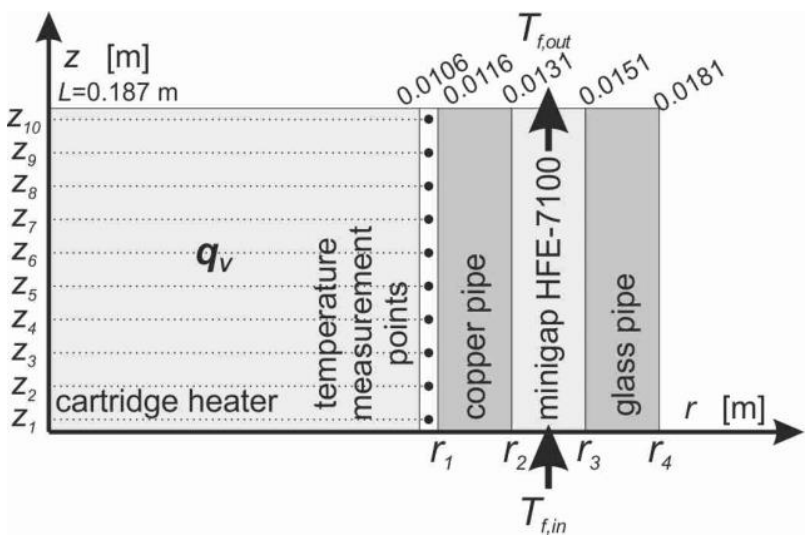

Fig. 3. Scheme of the test section (pictorial view, not to scale).

The temperature of the copper pipe $T_{c p}$ was assumed to satisfy Laplace's equation

$$
\frac{1}{r}\left(r \frac{\partial T_{c p}}{\partial r}\right)+\frac{\partial^{2} T_{c p}}{\partial z^{2}}=0
$$

for which we imposed proper boundary conditions: the layers of the cartridge heater and the copper pipe are in perfect thermal contact while the walls perpendicular to them are isolated, i.e.

$$
\begin{gathered}
\lambda_{c p} \frac{\partial T_{c p}}{\partial r}=\frac{q_{V} r_{1}}{2} \text { for } r=r_{1} \\
T_{c p}\left(r_{1}, z\right)=T_{a p p r o x}(z) \\
\left.\frac{\partial T_{c p}}{\partial z}=0 \text { for a }\right) z=0 \text { and b) } z=L
\end{gathered}
$$

where $\lambda_{c p}$ - the thermal conductivity of the cooper pipe, $L-$ length of the minigap, $q_{V}-$ volumetric heat flux 
supplied to the cartridge heater, $T_{\text {approx }}-$ a polynomial approximating the temperature measurements of the cooper pipe.

The IHCP described by equations (1) - (4) was solved by two methods, based on the Fourier transform in the first approach and on the Trefftz method in the second one.

The working fluid temperature $T_{f}$ was calculated depending on the flow type:

- for single phase flow and in the subcooled boiling region (small share of the vapour phase in the mixture), the fluid temperature changes linearly in the minichannel from the inlet temperature to the outlet temperature;

- for two-phase flow in the saturated boiling region (when it dominates the participation of the vapour phase in the mixture) - the fluid temperature is equal to local saturation temperature; local saturation temperature is determined on the basis of the linear distribution of pressure along the length of the minigap from the inlet to the outlet.

If the temperature distributions of the copper pipe and the refrigerant are known, we can calculate the heat transfer coefficient $\alpha$ at the fluid - pipe interface due to the Robin condition

$$
\alpha(z)=\frac{0.5 q_{V} r_{1}^{2} r_{2}^{-1}}{T_{c p}\left(r_{2}, z\right)-T_{f}(z)}
$$

\subsection{Fourier transform}

In the solution of IHCP described by equations (1) - (4) the Fourier transform was used, where the integraltransform $\hat{T}_{c p, F}$ and the inversion formula for the function $T_{c p, F}$ in the finite interval $0 \leq z \leq L$ were defined by the formulas respectively

$$
\begin{aligned}
& \hat{T}_{c p, F}\left(r, \beta_{i}\right)=\int_{0}^{L} T_{c p, F}(r, z) K\left(\beta_{i}, z\right) d z \\
& T_{c p, F}(r, z)=\sum_{i=0}^{\infty} \hat{T}_{c p, F}\left(r, \beta_{i}\right) K\left(\beta_{i}, z\right)
\end{aligned}
$$

The transform kernel $K$ is given by analogy to [14]

$$
K\left(\beta_{i}, z\right)=\left\{\begin{array}{cc}
\frac{1}{L} & \text { for } \quad i=0 \\
\sqrt{\frac{2}{L}} \cos \beta_{i} z & \text { for } \quad i=1,2, \ldots
\end{array}\right.
$$

and the eigenvalues $\beta_{i}=\pi i / L$ for $i=1,2, \ldots$.

The solution of equation (1) has the form

$$
T_{c p, F}(r, z)=\sum_{i=0}^{\infty}\left(a_{i} I_{0}\left(\beta_{i} r\right)+b_{i} K_{0}\left(\beta_{i} r\right)\right) K\left(\beta_{i}, z\right)
$$

where $a_{0}=\frac{1}{L} \int_{0}^{L} T_{\text {approx }}(z) d z, b_{0}=0, I_{0}-$ modified Bessel function of the first kind, $K_{0}$ - modified Bessel functions of the second kind. The coefficients $a_{i}$ and $b_{i}$ for $i=1,2, \ldots$, were calculated from the system of equations

$$
\left\{\begin{array}{l}
a_{i} I_{0}\left(\beta_{i} r_{1}\right)+b_{i} K_{0}\left(\beta_{i} r_{1}\right)=\int_{0}^{L} K\left(\beta_{i}, z\right) T_{\text {approx }}(z) d z \\
a_{i} \beta_{i} I_{0}\left(\beta_{i} r_{1}\right)+b_{i} \beta_{i} K_{0}\left(\beta_{i} r_{1}\right)=\frac{q_{V} r_{1}}{2 \lambda_{c p}} \int_{0}^{L} K\left(\beta_{i}, z\right) d z
\end{array}\right.
$$

The function $T_{c p}$ satisfies Laplace's equation (1) and the assumed boundary conditions (2)-(4) exactly.

\subsection{Trefftz method}

The idea of the Trefftz method consists in an approximation of an unknown solution of equation (1) using a linear combination of functions (called T-functions), which satisfy this equation exactly. First of all, T-function for the equation (1) should be defined. Solving Laplace's equation (1) by the Fourier method (separation of variables method), we obtain two solutions depends on Bessel functions of the first $J_{0}$ and second $Y_{0}$ kind

$$
\begin{array}{ll}
\text { a) } J_{0}(\theta r) e^{\theta z}, & \text { b) } Y_{0}(\theta r) e^{\theta z}
\end{array}
$$

Expanding the functions (11) into a power series in regard to powers of the $\theta$ parameter, yields T-functions of the first $u_{n}(x, y)$ and second $w_{n}(x, y)$ kind for equation, [15]

$$
\begin{gathered}
u_{n}(r, z)=\sum_{k=0}^{[n / 2]} \frac{(-1)^{k}}{(k !)^{2}(n-2 k) !}\left(\frac{r}{2}\right)^{2 k} z^{n-2 k} \\
w_{n}(r, z)=u_{n}(r, z) \cdot \ln r-\sum_{k=0}^{[n / 2]} \frac{(-1)^{n}(0.5 r)^{2 n} H_{n}}{(k !)^{2}(n-2 k) !} z^{n-2 k}
\end{gathered}
$$

where $H_{n}$ - harmonic numbers and the symbol [.] denotes the floor function.

Knowledge of the T-function given by equations (12) and (13) allows for approximating the unknown solution of Laplace's equation (1) in the form of a linear combination, [15]

$$
T_{c p, T}(r, z)=\sum_{n=0}^{N} c_{n} u_{n}(r, z)+\sum_{m=0}^{M} d_{m} w_{m}(r, z)
$$

Unknown coefficients $c_{n}, d_{m}$ in (14) were determined by minimizing the error functional that described the mean square error with which the function $T_{c p, T}$ satisfied the assumed boundary conditions (2)-(4), analogously as in [1]. The function $T_{c p, T}$ satisfies Laplace's equation exactly and the assumed boundary conditions (2)-(4) approximately. Approximate fulfillment of boundary conditions by the calculated approximation is a characteristic feature of the Trefftz method. 


\section{Results and discussion}

The calculations were performed with the Fourier transform and the Trefftz method for the following experimental parameters: pressure at the inlet $220 \mathrm{kPa}$ (average), inlet liquid subcooling $56 \mathrm{~K}$ (average), constant mass flow rate of $0.017 \mathrm{~kg} / \mathrm{s}$ ) and five heat fluxes $q_{w}$ in the range of $8.8 \div 17.4 \mathrm{~kW} / \mathrm{m}^{2}$.

Figure 4 shows the heat transfer coefficient as a function of the minigap inlet at the subcooled boiling region, calculated using the Fourier transform.

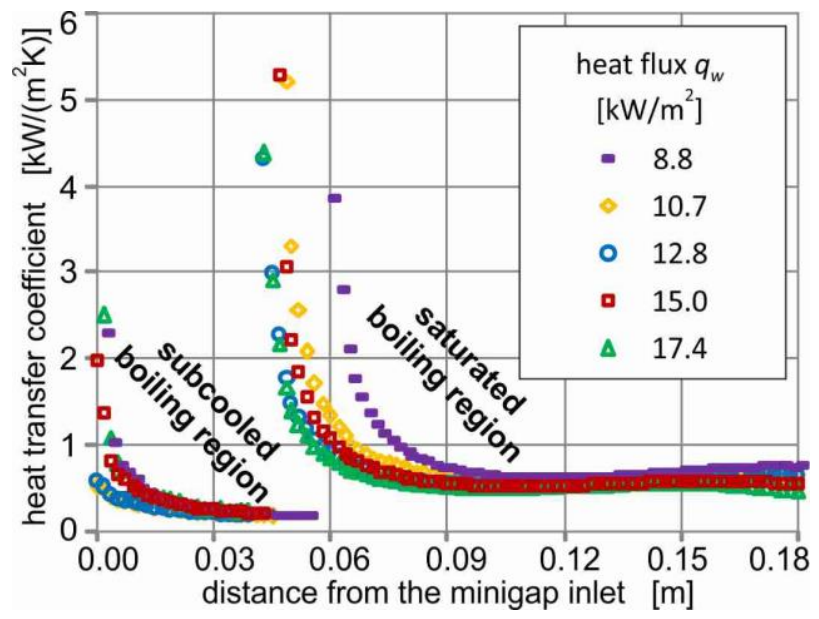

Fig. 4. The heat transfer coefficient $\alpha_{F}$ vs. the distance from the minigap inlet $z$.

For expected improvement of accuracy, measurement temperature of the cartridge heater - copper pipe interface was approximated with a third degree polynomial. In the Trefftz method the same number of T-functions of the first and second kind were taken. The gradient of the copper pipe temperature $T_{c p}$ was calculated based on the known foil temperature distribution.

Similar values of the heat transfer coefficient have been obtained based on the Trefftz method, which is confirmed by the values of the maximum relative differences. The maximum relative differences $M R D$ between heat transfer coefficient values obtained using the Trefftz method $\left(\alpha_{T}\right)$ and Fourier transform $\left(\alpha_{F}\right)$ were calculated from the formula

$$
M R D=\max \left\{\frac{\left\|\alpha_{T}-\alpha_{F}\right\|_{2}}{\left\|\alpha_{T}\right\|_{2}}, \frac{\left\|\alpha_{T}-\alpha_{F}\right\|_{2}}{\left\|\alpha_{F}\right\|_{2}}\right\}
$$

where $\mathrm{L}^{2}-$ norm is defined in in measurements points $z_{\text {data }}$ as

$$
\|\alpha\|_{2}=\sqrt{\sum_{z_{\text {data }}} \alpha^{2}\left(z_{\text {data }}\right)}
$$

For the considered heat fluxes, the $M R D$ ranges from 0.21 to 1.46 and only for heat flux $12.8 \mathrm{~kW} / \mathrm{m}^{2} M R D$ is equal to 12.10 .

By analogy to $[4,16,17]$, the mean relative error of the heat transfer coefficient (MRE) was determined only for the smallest and the largest value of heat flux. In the $M R E$ calculations it was adopted that:

- uncertainty of temperature measurement: $\Delta T_{\text {data }}=0.9 \mathrm{~K},[4]$,

- accuracy of the copper pipe temperature approximation: $\quad \Delta T_{c p}=\left|\frac{\partial T_{c p}}{\partial z} \Delta z\right|+\Delta T_{\text {data }} \quad$ (average:

$0.93 \mathrm{~K})$;

- uncertainty of thermocouple location: $\Delta z=10^{-4} \mathrm{~m}$,

- uncertainty of radius: $\Delta r=10^{-4} \mathrm{~m}$,

- accuracy of the volumetric heat flux supplied to the cartridge heater $\Delta q_{V}$ (average: $222 \mathrm{~kW} / \mathrm{m}^{3}$ ), [17];

- accuracy of the fluid temperature determination: $\Delta T_{f}=0.9 \mathrm{~K}$ in the subcooled boiling region and $\Delta T_{f}=\Delta T_{\text {sat }}$ in the saturated boiling region, [4].

Both used numerical methods gave the $M R E$ less than $9.4 \%$, except $q_{w}=12.8 \mathrm{~kW} / \mathrm{m}^{2}$, where $M R E$ was equal to $16.7 \%$ for the Fourier transform.

It was observed that local heat transfer coefficient values decreased with increasing distance from the minigap inlet at all heat fluxes supplied to the heater at the subcooled and the saturated boiling regions. In the subcooled boiling region, heat transfer coefficients were in several hundred $\mathrm{kW} /\left(\mathrm{m}^{2} \mathrm{~K}\right)$, which is much lower than those obtained for the saturated boiling region. The highest values of the heat transfer coefficient are in the order of several thousand $\mathrm{kW} /\left(\mathrm{m}^{2} \mathrm{~K}\right)$ and occur at the beginning of the saturated boiling region. The highest value reached $5.5 \mathrm{~kW} /\left(\mathrm{m}^{2} \mathrm{~K}\right)$.

It should be underlined that the heat transfer coefficient obtained with the use of the Fourier transform and by the Trefftz method were similar. Values of the maximum relative differences between results were in the range: $0.21-1.22$, the smallest values $M R D(0.21)$ were obtained for the smallest heat flux, for higher heat fluxes $M R D$ values were higher, and the highest one (1.22) was found for the highest heat flux.

$M R E$ was very similar for both used methods. The $M R E$ for Trefftz method did not exceed $9.4 \%$ in the entire experiment.

\section{Conclusions}

In this work, the application of two numerical methods for the identification of temperature distribution in flow boiling of HFE-7100 in an annular minigap was proposed. The two-dimensional temperature distribution of the copper pipe was determined from the Fourier transform and from the Trefftz method. Using the Fourier transform, a solution satisfying the governing differential equation and the adopted boundary conditions exactly. In the Trefftz method, the obtained approximation of the copper pipe temperature has been satisfying exactly only the governing differential equation - the boundary conditions are satisfied by the function approximately. Using the Trefftz method, as opposed to the Fourier transform, the IHCP may be solved also in regions with more complex shapes, with 
a lower number of boundary conditions, whether in a continuous form, or in a discrete form.

As the results of the mathematical modelling of the temperature distributions in the test section was known, the heat transfer coefficient at the fluid - wall interface was calculated due to the Robin condition. The results were presented as the heat transfer coefficient versus the distance from the minigap inlet. It was observed that local heat transfer coefficient values decreased with increasing distance from the minigap inlet at all heat fluxes supplied to the heater at the subcooled and the saturated boiling regions. In the subcooled boiling region, heat transfer coefficients were in several hundred $\mathrm{kW} /\left(\mathrm{m}^{2} \mathrm{~K}\right)$. The highest value in the order of several thousand $\mathrm{kW} /\left(\mathrm{m}^{2} \mathrm{~K}\right)$ occurred at the beginning of the saturated boiling region. The values of the heat transfer coefficient obtained with the use of the Fourier transform and by the Trefftz method were similar.

\section{References}

1. S. Hożejowska, M. Piasecka, Heat Mass Transf. 50, 1053-1063 (2014)

2. B. Maciejewska, M. Piasecka, Heat Mass Transf. 53(4), 1211-1224 (2017)

3. K. Strąk, M. Piasecka, B. Maciejewska, Int. J. Heat Mass Transf. 117, 375-387(2018)

4. B. Maciejewska, M. Piasecka, Int. J. Heat Mass Transf. 107, 925-933 (2017)

5. T. Musiał, M. Piasecka, S. Hożejowska, EPJ Web of Conf. 143, 02077 (2017)

6. M. Piasecka, S. Hożejowska, T. Musiał, 2017, E3S Web of Conf. 13, 02002 (2017)

7. M. Piasecka, S. Hożejowska, A. Piasecki, Proc. 9th World Conf. ExHFT-9, June 12-15, Brasil, PT30 (2017)

8. S. Hożejowska, T. Musiał, M. Piasecka, EPJ Web of Conf. 114, 02042 (2016)

9. G. Boye, J. Schmidt, F. Beyrau, Adv. Mech. Eng. 7 (6), 1-14 (2015)

10. F.C. Hsieh, K.W. Li, Y.M. Lie, C.A. Chen, T.F. Lin, Int. J. Heat Mass Transf. 51, 3763-3775 (2008)

11. C.P. Yin, Y.Y. Yan, T.F. Lin, B.C Yang, Int. J. Heat Mass Transf. 43, 1885-1896 (2000)

12. Z. Guo, B.S. Haynes, D.F. Fletcher, J. Comput. Mult. Flows 8, 61-82 (2016)

13. J. Yao, M.K. Patel, Y. Yao, P.J. Mason, Adv. Appl. Math. Mech. 1, 231-241 (2009)

14. M. N. Ozisik, Boundary Value Problems of Heat Conduction (International Textbook Company, Scranton, Pennsylvania, 1968)

15. S. Futakiewicz, The heat functions method for solving direct and inverse heat conduction problems, (Ph.D. Thesis in Polish, Poznan University of Technology, 1999)

16. M. Piasecka, Metrol. Meas. Syst. XX (2), 205-216 (2013)
17. S. Hożejowska, M. Piasecka, M.E. Poniewski, Int. J. Therm. Sci. 48, 1049-1059 (2009) 